\title{
Use of Venovenous Extracorporeal Membranous Oxygenation Following latrogenic Tracheal Rupture
}

Rajiv Kabadi, MD

\section{INTRODUCTION}

latrogenic tracheal rupture is a rare complication of endotracheal intubation and has a high risk of morbidity and mortality. Risk factors include female gender, short stature (height less than 160-cm), difficult airway anatomy, underlying connective tissue disorder, chronic obstructive pulmonary disease, use of a rigid stylet, inadequate intubation tube size, cuff over-inflation, emergent intubation and intubation performed by non-anesthesiologists. ${ }^{1}$ Early recognition is important and diagnosis requires bronchoscopic confirmation. ${ }^{2,3}$ We describe a case where emergent venovenous extra-corporeal membrane oxygenation ( $\mathrm{V}$-ECMO) was utilized in the management of tracheal rupture.

\section{CASE DESCRIPTION}

A 46-year-old female smoker with a history of asthma presented to an outside hospital with worsening dyspnea and was found to be in status asthmaticus. She underwent a difficult intubation on hospital day two for hypercapneic respiratory failure. She subsequently developed severe subcutaneous emphysema on her face that spread to her torso, arms and legs (Figure 1). Computed tomography of the chest revealed pneumothoraces, pneumomediastinum, pneumopericardium (Figure 2) and pneumoperitoneum (Figure 3). Bilateral chest tubes and a pericardial drain were placed and she was transferred to our facility for higher level of care.

Bronchoscopy was performed which revealed a 2-cm longitudinal laceration in the membranous wall of the trachea that extended towards the carina. Given these findings, cardiothoracic surgery was consulted. Due to refractory hypoxemic and hypercapnic respiratory failure despite maximal ventilatory support, VV-ECMO was initiated on hospital day three. Due to improvement in oxygenation and ventilation, a right thoracotomy with tracheal repair and pericardial window placement was performed.

The patient was continued on steroids and bronchodilators for management of her asthma exacerbation. V-ECMO was weaned and the patient underwent ECMO catheter decannulation on hospital day five. She underwent tracheostomy on hospital day fifteen with eventual decannulation prior to being discharged to acute rehab on hospital day thirty-one.
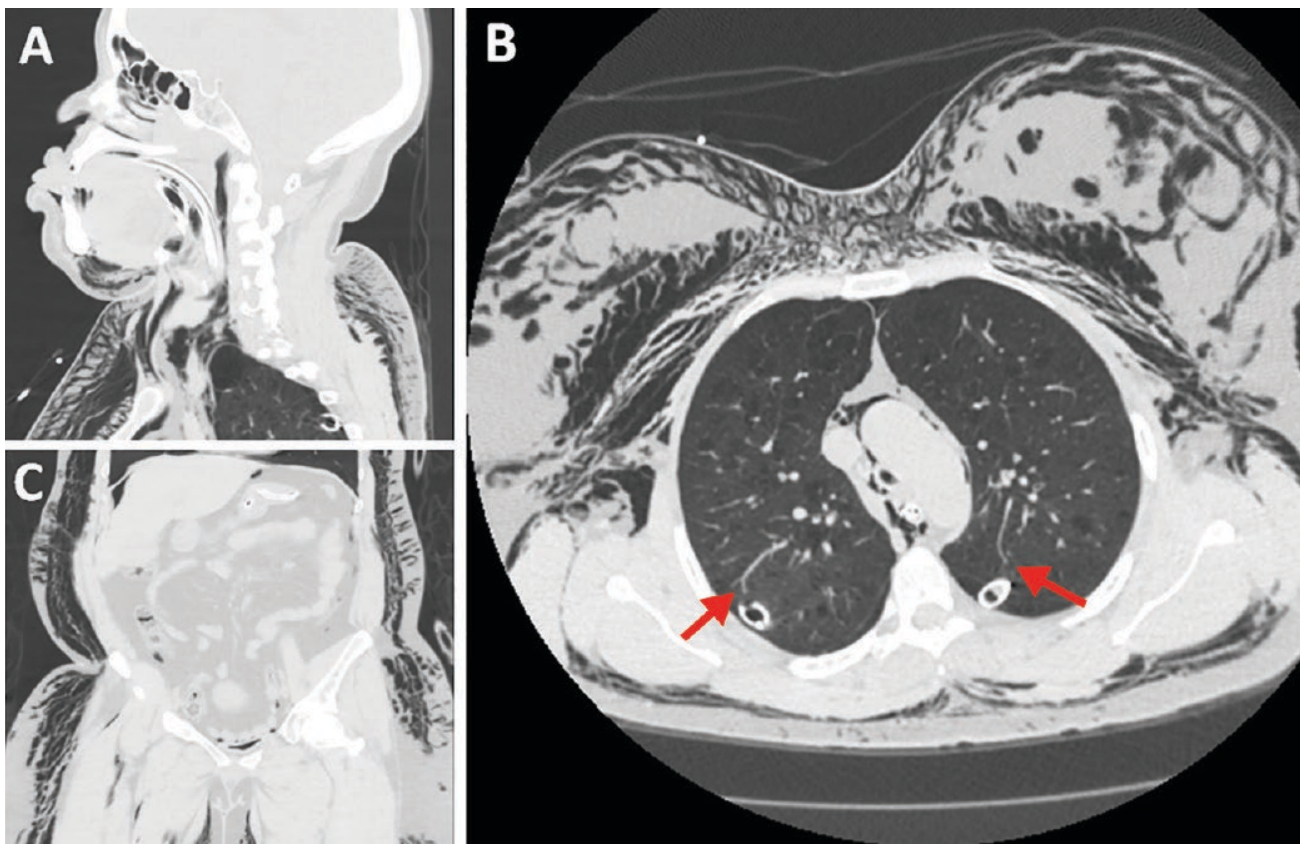

Figure 1. Computed tomographic (CT) imaging demonstrating extensive subcutaneous emphysema (A-C), apical bleb (A), emphysematous changes in the lungs (B) and bilateral chest tubes (B; arrows) 


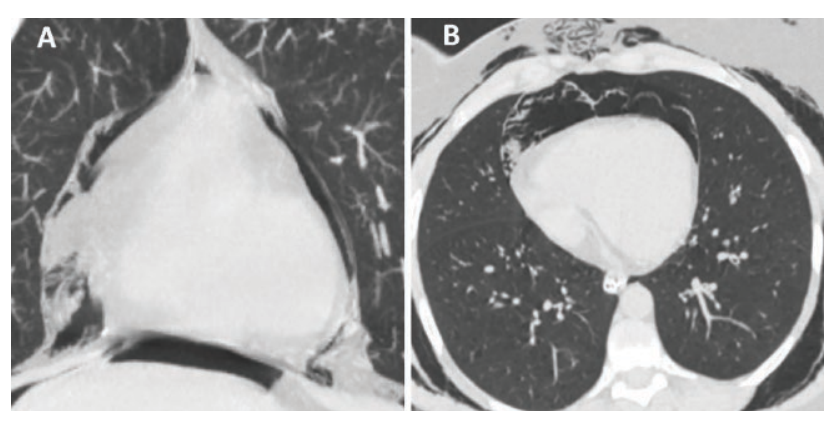

Figure 2. CT imaging demonstrating pneumomediastinum and pneumopericardium (A-B).



Figure 3. CT imaging demonstrating pneumoperitoneum (marked by red asterixis).

\section{OUTCOME AND FOLLOW UP}

The patient was last seen in the pulmonology clinic 6 months after she was discharged and has maintained good functional status. She was continued on standard care treatment for moderate persistent asthma which was well-controlled despite her continued cigarette smoking. Her major complaint at the recent office visit was anxiety rather than cardio-pulmonary symptoms.

\section{DISCUSSION}

Tracheal rupture is an uncommon but severe complication of endotracheal intubation. Conservative management should be considered for hemodynamically stable patients without sequela such as progressive air leak, sepsis, or esophageal injury. Non-operative strategies involve non-invasive positive-pressure ventilation for patients who are breathing spontaneously, or endotracheal intubation and fixation distal to the rupture site ("bridging") with low-tidal volume ventilation and permissive hypercapnia for patients with underlying respiratory disease. Additional measures include daily bronchoscopic examinations, post-pyloric feeding, limiting patient movement to specified times, and antibiotic therapy to reduce risks of mediastinitis and ventilator-associated pneumonia. ${ }^{4}$ Surgical intervention is reserved for patients with uncontrolled air leaks, active endobronchial hemorrhage, or for patients who have failed conservative measures as evinced by progressive ventilator requirements leading to extreme difficulties in oxygenation and ventilation as was the case for our patient.1,5 For our patient, VV-ECMO was used as a bridge to surgical tracheal repair in the setting of status asthmaticus and respiratory failure. Further studies should identify patients who will benefit from early surgical management and/or transfer to an ECMO-ready facility. ${ }^{3}$

\section{KEY POINTS}

- Tracheal rupture is an uncommon but severe complication of endotracheal intubation.

- VV ECMO may be used as a bridge to definitive management of tracheal rupture in patients with progressive respiratory failure who failed conservative strategies

- Further studies should identify patients who may benefit from early surgical management and/or transfer to a facility that has ECMO capabilities.

\section{REFERENCES}

1. Johnson AP, Cavarocchi NC, Hirose H. Ventilator strategies for WV ECMO management with concomitant tracheal injury and H1N1 influenza. Heart, Lung and Vessels. 2015;7(1):74-80

2. Miñambres E, Buron J, Ballesteros MA, et al. Tracheal rupture after endotracheal intubation: a literature systematic review. European Journal of Cardio-Thoracic Surgery. 2009:35(6):1056-1062.

3. Panagiotopoulos N, Patrini D, Barnard M, et al. Conservative versus Surgical Management of latrogenic Tracheal Rupture. Medical Principles and Practice. 2017:26:218-220.

4. Singh S, Gurney S. Management of post-intubation tracheal membrane ruptures: A practical approach. Indian Journal of Critical Care Medicine 2013:17(2):99-103.

5. Conti M, Pougeoise M, Wurtz A, et al. Management of Postintubation Tracheobronchial Ruptures. CHEST. 2006;130(2):412-418. 